\title{
Laparoscopic Gastrojejunostomy for the Treatment of SIRT-Induced Duodenal Ulcer Complicated by Gastric Outlet Obstruction
}

\author{
Walaa El Arja ${ }^{1,2}$ Sarah B. Eid ${ }^{2,3}$ Elias Saikaly 2,40 \\ ${ }^{1}$ Department of Gastroenterology, Saint George Hospital University \\ Medical Center, Beirut, Lebanon \\ 2 Faculty of Medicine, University of Balamand, North Governorate, \\ Lebanon \\ ${ }^{3}$ Department of General Surgery, Saint George Hospital University \\ Medical Center, Beirut, Lebanon \\ ${ }^{4}$ Department of Radiology, Saint George Hospital University Medical \\ Center, Beirut, Lebanon
}

Lynn Ezzeddine 2,4

Rayan Daoud ${ }^{2}$

Elias Fiani ${ }^{1}$

Address for correspondence Elias Fiani, MD, Saint George University, Saint George Hospital, Youssef Sursock Street, Beirut 166378,

Lebanon (e-mail: elias.fiani@hotmail.com).

Int J Recent Surg Med Sci 2022;8:63-65.

\begin{abstract}
Selective internal radiation therapy (SIRT) is an emerging therapeutic modality in patients with unresectable hepatocellular carcinoma or liver metastases. However, complications can occur due to migration of radiation microspheres such as gastrointestinal ulcer, cholecystitis, bleeding, pancreatitis, and many others. A 50year-old woman with stage IV breast cancer who underwent radioembolization for unresectable hepatic metastasis 6 months ago presented to our hospital with 1 month history of nausea, vomiting, with food intolerance, and weight loss. Esophagogastroduodenoscopy showed large deep duodenal bulbar ulcer along with antral ulcer-

Keywords

- duodenal ulcer

- gastric outlet obstruction

- gastrojejunostomy

- radioembolization

- yttrium-90 ations and edematous gastropathy. Biopsies revealed typical black, duodenal yttrium90 sphere, documenting radiation injury. After she was discharged on proton pump inhibitor, the patient came back 1 month later for exacerbation of symptoms; computed tomography scan of the abdomen showed gastric outlet obstruction. Although there is no consensus in treating radiation-induced ulcers, physicians should be aware of this complication in patient who underwent SIRT presenting for abdominal pain.
\end{abstract}

\section{Introduction}

Selective internal radiation therapy (SIRT) is an emerging therapeutic modality in patients with unresectable hepatocellular carcinoma or liver metastases. ${ }^{1,2}$ The procedure's rationale is that these tumors are vascularized by arterial blood flow, in contrast to normal hepatic parenchyma supplied by portal blood flow. ${ }^{1,3}$ Injecting $\beta$-emitting yttrium-90 $\left({ }^{90} \mathrm{Y}\right)$ microspheres into the hepatic arteries leads to necrosis of the lesion. ${ }^{4}$ However, complications have been described when radiation injury occurs in extrahepatic organs due to embolization of radioactive beads in other arteries. ${ }^{1}$ While constitutional symptoms (fever) are the most common adverse effects reported, ${ }^{5}$ more serious complications can occur, such as gastrointestinal ulcer, cholecystitis, bleeding, pancreatitis, hepatic decompensation, and radiation pneumonitis. ${ }^{2}$

Herein, we report a case of duodenal and gastric ulcers due to ${ }^{90} \mathrm{Y}$ seed migration complicated by gastric outlet
DOI https://doi.org/ $10.1055 / \mathrm{s}-0041-1736448$. ISSN 2455-7420.

\footnotetext{
(c) 2022. Medical and Surgical Update Society. All rights reserved. This is an open access article published by Thieme under the terms of the Creative Commons Attribution-NonDerivative-NonCommercial-License, permitting copying and reproduction so long as the original work is given appropriate credit. Contents may not be used for commercial purposes, or adapted, remixed, transformed or built upon. (https://creativecommons.org/ licenses/by-nc-nd/4.0/)

Thieme Medical and Scientific Publishers Pvt. Ltd., A-12, 2nd Floor, Sector 2, Noida-201301 UP, India
} 
obstruction to alert physicians of this rare and serious cause of gastric injury and to focus on management difficulties.

\section{Case Presentation}

A 50-year-old woman with stage IV breast cancer who underwent radioembolization for unresectable hepatic metastasis 6 months ago presented to our hospital with 1 month history of worsening nausea, vomiting, with food intolerance, and weight loss.

On presentation, she was hemodynamically stable, afebrile. On physical examination, she had a nondistended abdomen, positive bowel sounds, with mild epigastric tenderness. Digital rectal examination was negative for melena or fresh blood. Laboratory tests including complete blood count, liver function tests, creatinine, amylase, and lipase were unremarkable except for liver enzymes that were 1.5 times upper limit.

Computed tomography (CT) scan of the abdomen and pelvis showed diffuse thickening of gastric antrum and first portion of duodenum (-Fig. 1).

Esophagogastroduodenoscopy (EGD) showed large deep duodenal bulbar ulcer along with antral ulcerations and edematous gastropathy ( - Fig. 2A, B).

Histologic examination of duodenal biopsies showed very small round dark black spheres within the lamina propria of some capillaries, compatible with resin microspheres, impregnated with ${ }^{90} \mathrm{Y}$ radionuclide ( $\mathbf{- F i g}$. 3A, B), with innumerable pseudohyphae and spores compatible with candida spp, suggestive of ischemic duodenal ulcer with fungal colonization. Gastric biopsies showed chronic inflammation of the antral mucosa covered with candida.

She was started on intravenous continuous esomeprazole for 72 hours, then switched to twice daily for 4 weeks along with sucralfate $1 \mathrm{~g}$ oral gel for 14 days. She was discharged on liquid diet and scheduled for control EGD in 4 weeks.

To note, prior to radioembolization, hepatic, celiac, and superior mesenteric arteriograms were done. The left hepatic artery was seen to arise from the gastric artery. Prophylactic gastroduodenal coil embolization was performed without complication. Pre- and posttreatment scintigraphic

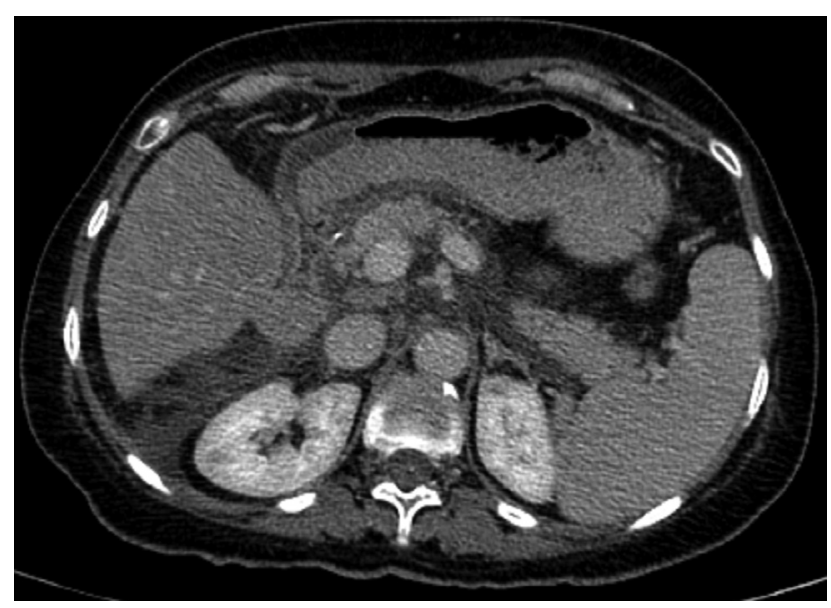

Fig. 1 Antral wall thickening on computed tomography scan.

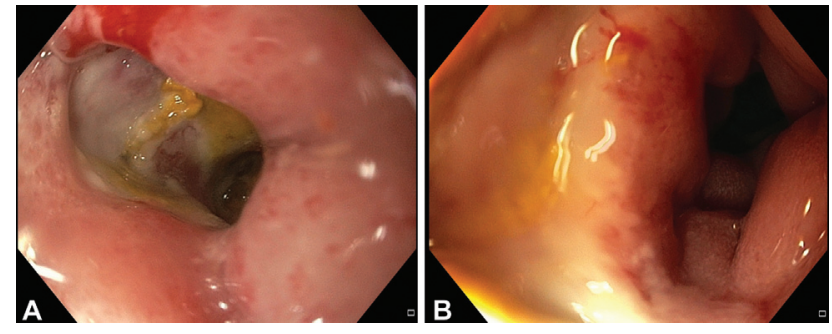

Fig. 2 Endoscopic view of (A) bulbar duodenal ulcer and (B) pyloric edema and erythema.
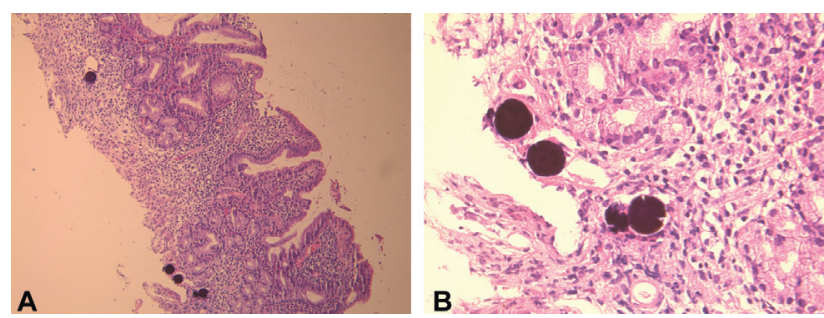

Fig. 3 (A) Low-power duodenal histology showing multiple black yttrium-90 spheres. (B) High-power histology showing a typical black, duodenal yttrium-90 sphere.

studies revealed no extrahepatic activity including no gastric uptake of microspheres.

One month later, the patient came back with exacerbation of symptoms: persistent nausea, vomiting, weight loss, and constipation.

CT scan of the abdomen at this time showed overdistension of the stomach with severe thickening of antrum and duodenal bulb, as well as near total collapse of distal duodenal portions (-Fig. 4A, B) in favor of gastric outlet obstruction.

Since the obstruction can be bypassed by the scope, and the mucosa was severely ulcerated and inflamed, laparoscopic gastrojejunostomy was therefore performed rather than endoscopic treatment. Five days postsurgery patient was discharged on proton pump inhibitor (PPI); her symptoms did not resolve completely, but she progressed in her diet gradually with better tolerance, and started to gain weight.

\section{Discussion}

SIRT utilizing ${ }^{90} \mathrm{Y}$ microspheres is an effective treatment modality for hepatic malignancies. The small size of these particles (20-30 $\mu \mathrm{m}$ in diameter) allows them to penetrate in
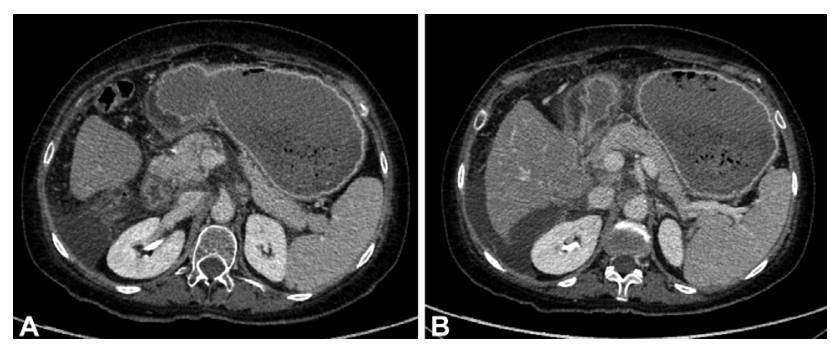

Fig. 4 Computed tomography (CT) scan showing (A) distended stomach with severe thickening of antrum and duodenal bulb and (B) near total collapse of distal duodenal portions. 
the microvasculature of the tumor supplied by hepatic artery, ${ }^{3}$ causing shrinkage of the malignancy by radioactivity effect of emitting isotope ${ }^{90} \mathrm{Y}$.

However, complications of this procedure can occur due to aberrant deposition or migration of radiation microspheres into nontarget locations, ${ }^{5,6}$ including normal hepatic parenchyma, pancreas, upper gastrointestinal tract, and lungs causing hepatitis, pancreatitis, ulcers, and pneumonitis. $^{4}$

The pathophysiology of the gastrointestinal ulcers results mainly from direct radiation mucosal injury in addition to ischemic injury due to mechanical arteriolar obstruction. ${ }^{1,4}$

Incidence of ulcerations decreased from $25 \%$ to less than $5 \%$ after strict adherence to contemporary protocols consisting of angiographic studies to assess the hepatic vasculature anatomy prior to procedure and to rule out any shunt. ${ }^{2}$ Median ulceration rate reported in previous studies was $8 \%$, ranging from 0 to $20 \%$, with $6 \%$ requiring surgical management mainly gastrectomy. ${ }^{2,7}$

Time to diagnosis varies from less than 1 month to over 9 months. ${ }^{2,6}$ The delay in diagnosis was mainly attributed to nonspecificity of symptoms consisting of nausea and vomiting.

These ulcers have proven to be difficult to treat and may be associated with severe abdominal pain, upper gastrointestinal, bleeding, bowel perforation, and obstruction. ${ }^{1}$

In our case, the duodenal ulcer was diagnosed 6 months after the procedure, localized in the bulb, rather than antrum and pylorus (commonly reported localizations). ${ }^{2}$

The anatomic variant of the left hepatic artery arising from left gastric artery (less commonly reported than the aberrant right hepatic artery $)^{2}$ may put this patient at risk for retrograde flow of the microspheres. ${ }^{6}$ Despite the preventive empiric coil embolization of the gastroduodenal artery, the patient had duodenal ulcer that may due to failure of detection of small vessels or to the development of new collaterals that occurs in 3 to $5 \%$ SIRT-induced gastrointestinal complications. ${ }^{1}$

Since microspheres were detected on biopsies, the patient was treated with PPI (high dose) like all the previous 51 reported cases ${ }^{1}$ in combination with sucralfate, but no response ( $45 \%$ of previous cases failed medical treatment ${ }^{1}$ ). The nonhealing could be attributed to ischemic pathophysiology of the ulcer, the superimposed infectious cause (candida), and the fact that these ulcers originate from the serosal surface, not from the mucosa, ${ }^{2,4}$ so not acid-related since parietal cells are mostly destructed by radiation. ${ }^{1}$ Antifungal treatment was not given, since no previous data recom- mended treatment of colonized ulcer and she had disturbed liver tests.

One month later, the symptoms worsened, SIRT-induced duodenal ulcer in our patient was complicated by gastric outlet obstruction, which imposed the decision to go for surgical management.

In all early studies, the procedures performed for refractory ulcers included distal gastrectomy (14\%), subtotal gastrectomy (14\%), partial gastrectomy (14\%), total gastrectomy (14\%), open small bowel resection with cholecystectomy and partial Billroth II gastrectomy (14\%), gastrectomy plus cholecystectomy (14\%), distal gastric resection, and Billroth I gastroduodenostomy (14\%). ${ }^{1,5}$

To the best of our knowledge, this is the first case whereby gastrojejunostomy is used to manage gastric outlet obstruction caused by SIRT-induced duodenal ulcer $^{1,5}$ with good clinical response.

In summary, despite the preventive measures taken prior to radioembolization, we still have evidence of gastroduodenal ulcer like our case that can be irreversible, difficult to treat, and can be associated with major complication like GOO.

\section{Conflict of Interest \\ None declared.}

\section{References}

1 Laila B, Vinciane L, Michael V, et al. Diagnosis, pathophysiology, and treatment of SIRT-induced gastroduodenal ulcers: a systematic literature review. Surg Oncol 2020;35:520-526

2 Yim SY, Kim JD, Jung JY, et al. Gastrectomy for the treatment of refractory gastric ulceration after radioembolization with $90 \mathrm{Y}$ microspheres. Clin Mol Hepatol 2014;20(03):300-305

3 Sacco R, Conte C, Tumino E, et al. Transarterial radioembolization for hepatocellular carcinoma: a review. J Hepatocell Carcinoma 2016;3:25-29

4 Voruganti IS, Godwin JL, Adrain A, Feller E. A woman with black beads in her stomach: severe gastric ulceration caused by Yttrium-90 radioembolization. Case Rep Med 2018;2018:1413724

5 Murthy R, Brown DB, Salem R, et al. Gastrointestinal complications associated with hepatic arterial Yttrium-90 microsphere therapy. J Vasc Interv Radiol 2007;18(04):553-561

6 Sjoquist KM, Goldstein D, Bester L. A serious complication of selected internal radiation therapy: case report and literature review. Oncologist 2010;15(08):830-835

7 South CD, Meyer MM, Meis G, et al. Yttrium-90 microsphere induced gastrointestinal tract ulceration. World J Surg Oncol 2008;6(01):93 